\title{
Extracto hidroalcohólico de cáscara de Citrus sinensis y aceite de Pinus patula, una alternativa de biorremediación
}

\author{
Hydroalcoholic extract of Citrus sinensis peel and Pinus patula oil, a bioremediation alternative
}

\author{
Marín-Velásquez Tomás (i)*, Barrutia-Barreto Israel
}

\begin{tabular}{l}
\hline Datos del Artículo \\
\hline Innova Scientificic SAC \\
Gerencia de Producción Científica. \\
Departamentod de Investigación. \\
Av. la Marina 1453. \\
San Miguel 15086 \\
Lima, Perú \\
Tel: (01)6768070/948631287. \\
Informes@innovascientific.com \\
ibarrutia@innovascientific.com \\
*Dirección de contacto: \\
Innova Scientific SAC \\
Gerencia de Producción Científica. \\
Departamento de Investigación. \\
Av. la Marina 1453. \\
San Miguel 15086 \\
Lima, Perú \\
Tel: (01)6768070/948631287. \\
Tomás Marín-Velásquez \\
E-mail address: tmarin@ @innovascientific.com \\
\hline \\
Palabras clave: \\
\hline Contaminación, \\
hidrocarburos, \\
impacto ambiental, \\
petróleo, \\
Pinus patula, \\
Citrus sinensis, \\
atenuación natural, \\
remediación, \\
suelo.
\end{tabular}

J. Selva Andina Res. Soc. 2020; 11(2):103-113. ID del artículo: 137/JSARS/2020

\section{Historial del artículo.}

\section{Recibido marzo 2020 \\ Devuelto mayo 2020. \\ Aceptado junio 2020. \\ Disponible en línea, agosto 2020.}

\begin{tabular}{c} 
Editado por: \\
Selva Andina \\
Research Society \\
\hline
\end{tabular}

\section{Keywords:}

Citrus sinensis,

hydrocarbons,

environmental impact,

envir
oil,

Pinus patula

pollution,

natural attenuation,

remediation,

soil.

\section{Resumen}

El objetivo de la investigación fue la evaluación del extracto hidroalcohólico de cáscaras de Citrus sinensis (ECN) y aceite de Pinus patula (ADP) como una alternativa de biorremediación, en una arena contaminada con combustible Diésel. Se contaminó una arena con combustible diésel en proporción $100 \mathrm{~mL} / \mathrm{kg}$ y se trató con los productos en dosis de 50 y $100 \mathrm{~mL} / \mathrm{kg}$ de arena contaminada, para luego monitorear la cantidad de extraíbles con hexano (HEM) cada 15 a 60 días. El diseño fue factorial de bloques al azar, con una muestra, una variable respuesta (HEM), tres factores (remediador, dosis y tiempo) y tres réplicas por bloque. Se utilizó ANOVA y test de Tukey para establecer la influencia de los factores experimentales sobre la variable respuesta. Se obtuvo que el tratamiento ECN logró una eficiencia de remediación máxima de $92.07 \%$ y para el ADP la eficiencia fue de $87.88 \%$, ambas superando a la eficiencia por atenuación natural que fue de $64.72 \%$. Se destaca que al tratar la arena contaminada con hidrocarburos con ECN y ADP se produce una mayor eficiencia de remediación respecto a la atenuación natural y la diferencia entre ambos tratamientos no fue estadísticamente significativa con un nivel de confianza de $95 \%$.

2020. Journal of the Selva Andina Research Society®. Bolivia. Todos los derechos reservados.

\section{Abstract}

The objective of the research was the evaluation of the hydroalcoholic extract of Citrus sinensis (ECN) peels and Pinus patula oil (ADP) as a bioremediation alternative, in a sand contaminated with diesel fuel. A sand was contaminated with diesel fuel in a proportion of $100 \mathrm{~mL} / \mathrm{kg}$ and the products were treated in doses of 50 and 100 $\mathrm{mL} / \mathrm{kg}$ of contaminated sand, to then monitor the amount of hexane extractables (HEM) every 15 to 60 days. The design was a random block factorial, with a sample, a response variable (HEM), three factors (remediator, dose and time) and three replicates per block. ANOVA and Tukey's test were used to establish the influence of experimental factors on the response variable. It was obtained that the ECN treatment achieved a maximum remediation efficiency of $92.07 \%$ and for ADP the efficiency was $87.88 \%$, both surpassing the natural attenuation efficiency that was $64.72 \%$. It is highlighted that treating hydrocarbon-contaminated sand with ECN and ADP produces a greater remediation efficiency compared to natural attenuation and the difference between both treatments was not statistically significant with a confidence level of $95 \%$. 


\section{Introducción}

La contaminación del suelo con hidrocarburos en un problema común, no solo en países productores de petróleo, también ocurre en todo el mundo, debido a los derivados del petróleo (DP) que se utilizan como combustible y lubricantes a nivel global ${ }^{1}$. La contaminación por hidrocarburos puede ocurrir por descargas de efluentes contaminados, fugas en tanques de almacenamiento o pérdidas accidentales en puntos de distribución ${ }^{2}$. La introducción de los hidrocarburos en el suelo genera la modificación sus condiciones de equilibrio natural, modificando sus propiedades físicas, químicas y biológicas ${ }^{3}$. El suelo está constituido por cinco componentes principales, la matriz mineral, aire, agua, materia orgánica y biota $^{4}$. Los hidrocarburos impiden el desarrollo normal de la vida en el suelo, al modificar el equilibrio vital entre estos elementos, generando un impacto de importancia al impedir el intercambio gaseoso con la atmósfera, lo que genera una serie de procesos físico-químicos como la volatilización y la penetración que en conjunto con las características del suelo generan un mayor o menor impacto ${ }^{5,6}$.

Existen diferentes tecnologías para la remediación de suelos contaminados, sin embargo el uso de microrganismos (biorremediación) es la más utilizada en caso de contaminación por hidrocarburos, debido a que ésta tiene como bondad el uso de los procesos microbiológicos naturales en el suelo, a través de ellos logra la degradación del contaminante ${ }^{7}$. La biorremediación de los hidrocarburos en el suelo se fundamenta en la premisa de que la presencia de hidrocarburos incrementa la actividad microbiana, ya que los microorganismos los utilizan como fuente de carbono y energía para el desarrollo de sus procesos metabólicos, lo que induce a su degradación ${ }^{8}$. Entre las técnicas biológicas, la bioestimula ción, que consiste en adicionar al suelo contaminado nutrientes que aumenten la actividad microbiana, siendo la más utilizada debido a que los microorganismos autóctonos producen mejores y más rápidos resultados 9 .

Las bondades de la bioestimulación como técnica para la remediación de suelos contaminados con hidrocarburos ha sido estudiada en diversas investigaciones, que han justificado su eficiencia ${ }^{10-13}$. Se ha comprobado que el uso de nutrientes de origen natural es efectiva para la bioestimulación de los microorganismos, por lo que en la presente investigación tuvo como objetivo evaluar el extracto de cáscara de naranja (Citrus sinensis) y el aceite de pino (Pinus patula) como bioestimulantes, midiendo su efecto sobre la degradación de los hidrocarburos constituyentes del diésel de petróleo.

\section{Materiales y métodos}

En la investigación se trabajó con una arena obtenida de una cantera de extracción de materiales para construcción, la cual fue caracterizada a través del método de tamizado para obtener su perfil granulométrico, según el protocolo de ensayo descrito en el método de prueba estándar para el análisis del tamaño de las partículas de los suelos ${ }^{14}$. También se determinó el porcentaje de extraíbles con hexano $(\mathrm{HEM}){ }^{15}$ y el $\mathrm{pH}$ mediante el método de prueba estándar para el $\mathrm{pH}$ de los suelos ${ }^{16}$.

Los productos remediadores utilizados fueron el extracto de cáscaras de naranja ( $C$. sinensis), se obtuvo a partir de material fresco que se fraccionó en pedazos pequeños $(1 \mathrm{~cm})$ se lavó y secó al sol por cinco días, luego se colocó en extractores Soxhlet con una mezcla de alcohol isopropílico y agua 
(70:30). El extracto fue concentrado en un rotaevaporador, se preparó una solución al $5 \%$ en agua ${ }^{17}$. El aceite de pino se obtuvo de proveedores comerciales (Macroquímicos S.A.C).

La arena fue caracterizada mediante análisis granulométrico $^{14}$, posteriormente fue contaminada con combustible diésel comercial (DB2), utilizando 100 $\mathrm{mL} / \mathrm{kg}$ de arena $(79000 \mathrm{mg} / \mathrm{kg})$, se dejó en reposo por siete días a temperatura promedio de $25{ }^{\circ} \mathrm{C}$. Se aplicó cada remediador: extracto de cáscaras de naranja (ECN) y aceite de pino (ADP) en dos dosis (50 y $100 \mathrm{~mL} / \mathrm{kg}$ de arena contaminada) y se realizó un monitoreo de la variable HEM a los 7, 15, 30, 45 y 60 días. Cada tratamiento se realizó por triplicado, lo que generó un diseño factorial con una variable respuesta (HEM) y 3 factores experimentales (Tipo de remediador (TR), Dosis aplicada (DA) y Tiempo (T)). El diseño seleccionado fue de una muestra con tres factores experimentales, una unidad control y tres réplicas, para un total de 96 ejecuciones, con una muestra tomada en cada ejecución. Las unidades experimentales (UE) se distribuyeron de forma aleatoria y se mantuvieron a condiciones controladas de laboratorio (temperatura promedio de $25^{\circ} \mathrm{C}$, con $6 \mathrm{~h}$ de luz diarias y humedad promedio de $80 \%)$.

Se calculó la eficiencia de degradación de cada uno de los tratamientos aplicados, así como de la atenuación natural (SR) (unidad control) mediante la ecuación $1^{11}$

Los resultados fueron tratados estadísticamente mediante análisis factorial, se realizó previamente una prueba de normalidad mediante los test de $\mathrm{Kol}$ mogorov-Smirnov y Shapiro-Wilks ${ }^{18}$ para corroborar que si los datos se ajustan a una distribución normal, para luego aplicar una prueba ANOVA factorial con contraste de rangos de Tukey ${ }^{19}$, para establecer si los factores experimentales muestran influencia 105 significativa sobre la variable dependiente. El nivel de confianza utilizado fue de 95\% $(\alpha=0.05)$. Se utilizó para los cálculos el software estadístico InfoStat versión $2018^{20}$.

\section{Resultados}

\section{Figura 1 Curva granulométrica de la muestra de Arena}

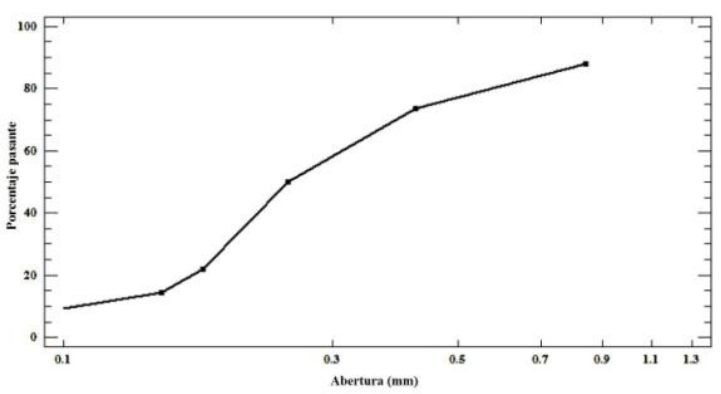

Se observa una predominancia de granos de mayor tamaño, es decir el material que logró pasar a través de las aperturas mayores, por lo que se obtuvo que los granos de arena entre 0.063 a $2 \mathrm{~mm}$ representaron el $94.35 \%$ y $5.65 \%$ corresponde a partículas de limo, no se observó partículas de arcilla $(<0.04$ $\mathrm{mm})$. De acuerdo a los resultados, se trabajó con una muestra de arena fina, en función a su ubicación en el triángulo textural, basado en la hoja de cálculo USDA $^{21}$. El pH se obtuvo un valor 6.0 medido en una proporción 1:2.5 clasificando a la arena como moderadamente ácida.

La arena se contaminó con una proporción de 100 $\mathrm{mL}$ de diésel $/ \mathrm{kg}$, que representa un porcentaje inicial de HEM adicionado de $7.88 \%$, valor obtenido luego de determinar la densidad del diésel, de 0.855 $\mathrm{g} / \mathrm{mL}$. Luego de siete días en reposo, se determinó el HEM de la arena obteniéndose un valor de $6.52 \%$, que representa una disminución de $17.3 \%$, se debe a la SR y la volatilización de los hidrocarburos más ligeros del derivado de petróleo, que tiene un rango de componentes alifáticos entre $\mathrm{C}_{15} \mathrm{y}$ 
$\mathrm{C}_{18}{ }^{22}$. Si se aplica el tratamiento desde el primer día se incurre en el error de contabilizar esta pérdida de hidrocarburos como parte del proceso de biorreme- diación, ya que el proceso de vaporización de los hidrocarburos es parte de la degradación por procesos naturales ${ }^{23}$.

Tabla 1 Resultados promedio de porcentaje de aceite y grasas en relación al tiempo

\begin{tabular}{cccc}
\hline Tiempo (Días) & Tipo de Remediador & Dosis aplicada $(\mathbf{m L})$ & HEM $(\%)$ \\
\hline \multirow{3}{*}{15} & SR & 0 & 5.30 \\
& ECN & 50 & 3.41 \\
& ECN & 100 & 4.91 \\
& ADP & 50 & 2.95 \\
& ADP & 100 & 3.66 \\
\hline SR & 0 & 4.10 \\
& ECN & 50 & 2.61 \\
& ECN & 100 & 3.57 \\
& ADP & 50 & 2.35 \\
& ADP & 100 & 2.73 \\
\hline \multirow{3}{*}{45} & SR & 0 & 3.23 \\
& ECN & 50 & 1.16 \\
& ECN & 100 & 1.23 \\
& ADP & 50 & 1.16 \\
& ADP & 100 & 1.24 \\
\hline SR & 0 & 2.78 \\
& ECN & 50 & 0.63 \\
& ECN & 100 & 0.62 \\
& ADP & 50 & 0.96 \\
& ADP & 100 & 0.95 \\
\hline
\end{tabular}

SR: Arena contaminada sin remediación, ECN: Extracto de cáscaras de naranja, ADP: Aceite de pino.

Figura 2 Comportamiento de los valores de HEM en función al tiempo

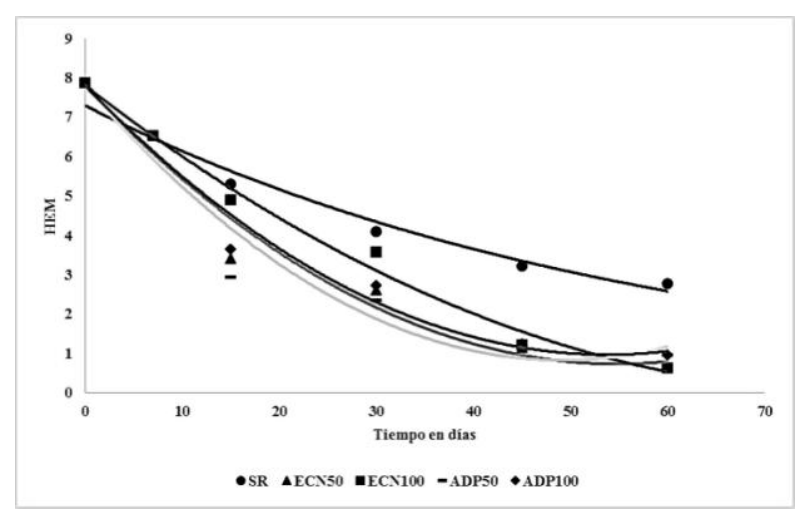

Se observa como las muestras tratadas con los remediadores presentan una disminución de HEM mayor a los valores por SR. Se destaca el comportamiento del tratamiento ECN con $100 \mathrm{~mL}$, a pesar que al inicio (15 días) presentó un HEM cercano al arrojado por SR, reportando una disminución marcada hasta llegar a valores cercanos a los de los otros tratamientos al cabo de los 60 días de prueba. El cálculo de la eficiencia de degradación (ecuación 1) dio como resultado que el remediador con la mayor eficiencia fue el ECN con resultados similares para las dos dosis aplicadas de $92.07 \%$ en promedio respecto al valor inicial. Igualmente se obtuvo para el ADP una eficiencia de $87.88 \%$, resultó menor a la obtenida para ECN. A pesar de observarse una tendencia a la SR, la eficiencia en este caso fue de $64.72 \%$, valor menor a los obtenidos con los tratamientos. Además se observó que según la tendencia de los resultados para que la unidad control alcance el valor final menor de HEM obtenido con los tratamientos $(0.95 \%)$, se debe esperar aproximadamente 60 días más, es decir el doble de los días 
requeridos para remediar la arena tratada al aplicar los productos evaluados.

El tratamiento estadístico de los datos inició con los test de normalidad de los valores obtenidos de HEM mediante Kolmogorov-Smirnov y Shapiro-Wilks, con la finalidad de determinar si los datos obtenidos presentan una distribución normal, lo que condiciona el tipo de estadística a aplicar (paramétrica o no paramétrica $)^{18}$. En el primer test se obtuvo que el p- valor mayor al nivel de significancia establecido de $\alpha=0.05$ ( $\mathrm{p}$-valor $=0.2644$ ), lo que indicó que los datos se ajustan a una distribución normal, el segundo test arrojó un p-valor=0.067, mayor a 0.05.

Los resultados anteriores indican que los datos de HEM se ajustan a una distribución normal, por lo tanto, se pueden aplicar pruebas estadísticas paramétricas, en este caso el ANOVA factorial y el contraste de rangos de Tukey.

Tabla 2 Resultado de análisis de varianza multifactorial para HEM

\begin{tabular}{llllll}
\hline Factores & SC & gl & CM & F & p-valor \\
\hline Tiempo (Días) & 555.60 & 5 & 111.12 & 865.46 & $<0.0001$ \\
Dosis (ml) & 38.02 & 2 & 19.01 & 148.08 & $<0.0001$ \\
Tipo de Remediador & 0.43 & 1 & 0.43 & 3.34 & 0.0712 \\
Error & 11.17 & 87 & 0.13 & & \\
Total & 605.22 & 95 & & & \\
\hline
\end{tabular}

Los resultados en la Tabla 2 indican que los factores Tiempo y Dosis ejercieron un efecto estadísticamente significativo sobre la variable HEM (p-valor <
0.05) y el factor TR no fue significativo sobre los resultados por presentar un $\mathrm{p}$-valor $>0.05$.

Tabla 3 Contraste de rangos de Tukey para HEM por Tiempo (Días)

\begin{tabular}{clllllll}
\hline Tiempo (Días) & Medias & n & E.E & Contrastes & & \\
\hline 60 & 1.43 & 16 & 0.09 & $\mathrm{~A}$ & & & \\
45 & 1.85 & 16 & 0.09 & & $\mathrm{~B}$ & & \\
30 & 3.28 & 16 & 0.09 & & $\mathrm{C}$ & $\mathrm{D}$ & \\
15 & 4.27 & 16 & 0.09 & & & $\mathrm{E}$ & $\mathrm{F}$ \\
7 & 6.52 & 16 & 0.09 & & & \\
0 & 7.88 & 16 & 0.09 & & $\mathrm{DMS}=0.369$ & \\
\hline
\end{tabular}

La Tabla 3, los resultados de HEM son estadísticamente diferentes en cada intervalo de tiempo en el que fueron medidos, lo que indica que, en el lapso de tiempo de la investigación, no se estabilizó el valor de la cantidad de hidrocarburos en la arena, sino que aun a los 60 días seguía disminuyendo de forma significativa.

Tabla 4 Contraste de rangos de Tukey para HEM por Dosis $(\mathrm{mL})$

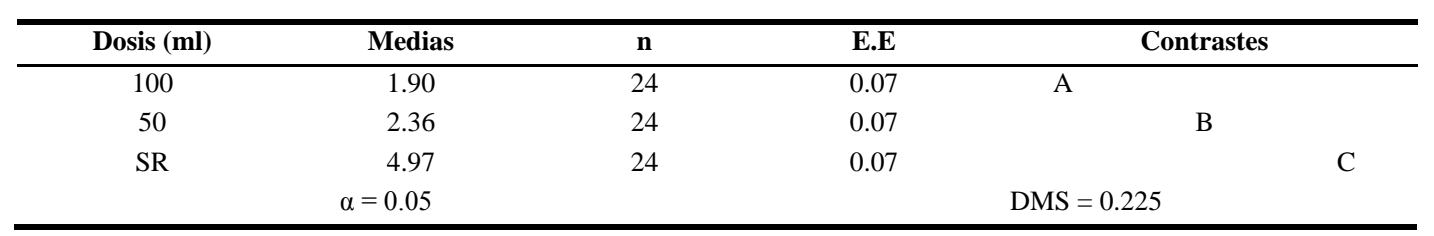


La Tabla 4 se observan diferencias significativas con un nivel de significancia de 0.05 entre las dos dosis aplicadas, así como entre éstas y los resultados obtenidos en la unidad control a la que no se le aplicó ningún tratamiento.

Tabla 5 Contraste de rangos de Tukey para HEM por Tipo de Remediador

\begin{tabular}{|c|c|c|c|c|}
\hline Tipo de Remediador & Medias & n & E.E & Contrastes \\
\hline ADP & 3.28 & 48 & 0.05 & $\mathrm{~A}$ \\
\hline \multirow[b]{2}{*}{$\alpha=0.05$} & 3.41 & 48 & 0.05 & A \\
\hline & & & $\mathrm{DM}$ & \\
\hline
\end{tabular}

La Tabla 5 se observa que, al aplicar los dos agentes remediadores estudiados, a pesar de existir una diferencia en la media de los resultados que favorece al ADP, esta diferencia no es estadísticamente significativa.

\section{Discusión}

$\mathrm{Al}$ analizar la muestra de arena, no se obtuvo HEM en la muestra, lo que indica que, al ser una arena limpia, no presentó material con aceites solubles en n-hexano, por lo que se puede decir que cualquier presencia de aceites (hidrocarburos) posteriores se debe exclusivamente a una adición externa (producto de la contaminación).

El análisis factorial realizado mostró que los factores DR y TR son determinantes en los resultados (tabla 2). Se observó que a mayor dosis aplicada, mayor es la eficiencia de degradación de los hidrocarburos, este es un resultado que se ha observado en otra investigación ${ }^{11}$, que utilizó como remediador el extracto de fibra de coco, la concentración utilizada influyó directamente en el resultado al igual que en la presente investigación, observándose porcentajes de eficiencia similares de $90.14 \%$. La influencia del tiempo de remediación, es una tendencia esperada, debido a que mientras más tiempo están los hidrocarburos en el suelo, su cantidad va disminuyendo por la acción de los procesos fisicoquímicos y biológicos que los degradan ${ }^{1,24,25}$.
Se observó, que la incorporación de extractos y aceites vegetales influyen significativamente en la velocidad de degradación de los hidrocarburos en una muestra de arena y se logra una disminución entre 87.88 y $92.97 \%$. Los sólidos residuales del procesamiento de la caña de azúcar, también se han utilizado como remediadores, los que influyen de forma significativa en la degradación de los hidrocarburos $^{26}$. Así, al aplicar sustratos orgánicos a un suelo contaminado con petróleo, también se obtuvieron resultados satisfactorios, con degradación de hidrocarburos entre 55 y $60.1 \%$, valores que fueron superados en la presente investigación ${ }^{27}$. Igualmente, el ECN fue utilizado para remediar un suelo contaminado con petróleo, y manifestar su influencia en la velocidad de degradación de los hidrocarburos, lo que concuerda con los resultados de la presente investigación resultados ${ }^{12,17}$.

El AP, puede ser utilizado como remediador con resultados que son estadísticamente similares a los obtenidos con ECN, sin embargo no se observaron investigaciones similares, para que nuestros resultados puedan sir motivo de contrastación, aunque el uso de biomasa de pino se ha utilizado en la preparación de compost y aplicado como enmienda en el proceso de biorremediación ${ }^{28}$. La acción del AP como remediador se explica por su composición, una mezcla de hidrocarburos terpénicos y alcoholes terpénicos cíclicos, solventes de los hidrocarburos, además de ser utilizados para la extracción de acei- 
tes $^{29,30}$. Este efecto de disolución de los hidrocarburos hace que estén biodisponibles para ser utilizados por microorganismos del suelo, lo que permite que acelere su degradación. El aceite de agujas de pino (Cedrus deodara) presento propiedades antimicrobianas, se puede pensar en una contradicción respecto a los resultados obtenidos, sin embargo, se usó el aceite sobre cepas de bacterias degradadoras de alimentos y bajo condiciones de laboratorio, sin la presencia de hidrocarburos ${ }^{31}$, por lo tanto, no se pueden comparar los resultados. También, se ha estudiado la actividad antimicrobiana del aceite de Pinus halepensis sobre cepas de Escherichia coli, en cultivos in vitro ${ }^{32}$ y se señaló su efectividad, sin embargo, la aplicación fue directa, bajo condiciones muy diferentes a las establecidas en la presente investigación, por lo que se debe seguir investigando sobre el efecto del AP como remediador.

La composición del ECN es mayoritariamente hidrocarburos terpénicos principalmente el limoneno ${ }^{33,34}$, por lo que el extracto hidroalcohólico obtenido posee estos componentes, además de otros, solubles en agua como los flavonoides, principalmente la naringina, ${ }^{35}$ lo que hace que sus componentes principales sean similares a los del AP, esto influyó en que en el análisis factorial y el test de Tukey se determinara que ambos remediadores actúan de manera igual desde el punto de vista de la estadística ( $p$-valor > 0.05). Se puede afirmar entonces que bajo las condiciones de esta investigación, el proceso de remediación de la arena estuvo influenciado por la dosis de agente remediador utilizado y el tiempo que se dejó actuar y no por el tipo de remediador aplicado.

Al comparar los resultados con los de la SR, se observó que aunque es evidente que la arena puede remediarse sin el uso de la remediación, la aplicación de esta técnica puede reducir el tiempo del 109 proceso, ya que en el lapso estudiado se alcanzó un máximo de eficiencia de $64.72 \%$ y para lograr llegar a la máxima eficiencia obtenida con los tratamientos se necesitarían 60 días más, lo que representa el doble de los utilizados en la investigación. La eficiencia de la remediación de suelos contaminados con hidrocarburos comparada con la SR también fue estudiada en una investigación previa ${ }^{13}$, sin embargo en la misma, la diferencia entre la el tratamiento y la SR fue menor en comparación a los resultados obtenidos en la presente investigación, esto se debe principalmente a que se utilizaron suelos diferentes y se sabe que el tipo de suelo y sus características son determinantes en la remediación ${ }^{36-38}$. La degradación de diésel en el suelo por SR y remediación con productos comerciales, también fue estudiada previamente $^{39}$, con resultados que coinciden con los obtenidos en la presente investigación, con lo que se concluye, que la incorporación de los productos remediadores aumenta el porcentaje de degradación de forma significativa y se demuestra su potencial aplicabilidad.

Los resultados obtenidos en esta investigación manifestaron que la remediación mediante la aplicación del ECN (C. sinensis) y AP (P. patula) es satisfactoria para disminuir los HEM presentes en una arena contaminada con diésel, observándose que ambos agentes remediadores actúan de forma similar, según el análisis ANOVA $(\alpha=0.05)$. Se observaron diferencias significativas respecto a las dosis de bioestimulante aplicadas, así como también respecto a la cantidad de aceites y grasas en función al tiempo.

Lo anterior indica que la cantidad de remediador aplicado a la muestra de arena contaminada influye de manera significativa en el proceso de remediación y que su aplicación mejora el proceso de SR. 
El uso de remediadores de origen vegetal como los utilizados, son una alternativa viable para la biorremediación de arenas contaminadas con diésel, según los resultados obtenidos, por lo que se debe seguir investigando la aplicación de los mismos en arenas contaminadas con otros tipos de derivados del petróleo como la gasolina, queroseno o aceites lubricantes, así como estudiar la eficiencia de los bioestimulantes utilizados aplicados a suelos de diferentes texturas.

\section{Fuente de financiamiento}

La investigación fue financiada por Innova Scientific SAC Perú.

\section{Conflictos de intereses}

Los autores declaran que no existe ningún tipo de conflictos de interés derivados de la realización de la presente investigación.

\section{Agradecimientos}

Los autores agradecen a la empresa Innova Scientific SAC Perú por todo el apoyo dado para la realización de la investigación.

\section{Aspectos éticos}

La aprobación de la investigación por el Comité de Ética, Centro de Investigación Científica Innova Scientific Perú y siguió las pautas establecidas para este comité.

\section{Literatura citada}

1. Velásquez Arias JA. Contaminación de suelos y aguas por hidrocarburos en Colombia. Análisis de la fitorremediación como estrategia biotecnológica de recuperación. Rev Investig Agrar Ambient 2017;8(1):151-67. DOI: https://doi.org/ 10.22 490/21456453.1846

2. Verma S, Bhargava R, Pruthi V. Oily sludge degradation by bacteria from Ankleshwar, India. Int Biodeterior Biodegradation 2006;57(4):20713. DOI: https://doi.org/10.1016/j.ibiod.2006.02. $\underline{004}$

3. Truskewycz A, Gundry TD, Khudur LS, Kolobaric A, Taha M, Aburto Medina A, et al. Petroleum hydrocarbon contamination in terrestrial ecosystems-fate and microbial responses. Molecules 2019;24(18):3400-20. DOI: https:// doi.org /10.3390/molecules24183400

4. Volke Sepúlveda T, Velasco Trejo JA, editores. Tecnologías de remediación para suelos contaminados [Internet]. México: Instituto Nacional de Ecología; 2002 [citado 22 de octubre de 2019]. 62 p. Recuperado a partir de: https://www. researchgate.net/publication/31851896 Tecnologias de remediacion para suelos contaminados _T_Volke_Sepulveda_JA_Velasco_Trejo

5. Adams Schroeder RH, Domínguez Rodríguez VI, García Hernández L. Potencial de la biorremediación de suelo y agua impactados por petróleo en el trópico mexicano. Terra Latinoam 1999;17(2):159-74.

6. Sivakumaran S, Robinson B, Mills T, Vogeler I, Clothier B, Northcott GL, et al. Bioremediation of soils contaminated with organic compounds. In: Sivakumaran S, Robinson B, Mills T, Vogeler I, Clothier B, Northcott GL, et al, editors. Australian New Zealand Soils Conference: 5, 6, 7, 8 y 9 december 2004. University of Sydney, Australia [Internet]. Australia: The Regional Institute online Publishing; 2004 [citado 21 de junio de 2020]. p. 1-6. Recuperado a partir de: file:/// 
C:/Users/usuario/Downloads/1455 sivakumarans \%20(1).pdf

7. Sims RC. Site characterization requirements. In: Seminars Bioremediation of Hazardous Waste Sites: Practical Approaches to Implementation: April 1993. Office of Research and Development [Internet]. Washington Environmental Protection Agency; 1993 [citado 3 de mayo de 2019]. p. 119. Recuperado a partir de: https://nepis. epa.gov/Exe/tiff2png.cgi/300023TV.PNG?$\mathrm{r}+75+-\mathrm{g}+7+\mathrm{D} \% 3 \mathrm{~A} \% 5 \mathrm{CZYFILES} \% 5 \mathrm{CINDEX} \%$ 20DATA\%5C91THRU94\%5CTIFF\%5C000006 57\%5C300023TV.TIF

8. Head IM, Jones DM, Larter SR. Biological activity in the deep subsurface and the origin of heavy oil. Nature 2003;426(6964):344-52. DOI: https:// doi.org/10.1038/nature02134

9. Schloter M, Dilly O, Munch JC. Indicators for evaluating soil quality. Agric Ecosyst Environ 2003;98(1-3):255-62. DOI: https://doi.org/10. 1016/S0167-8809(03)00085-9

10.Islas García A, Peralta Rodríguez M, Vega Loyo L, López Aguilar R, Rodríguez Vázquez R. Biorremediación por bioestimulación y bioaumentación con microorganismos nativos de un suelo agrícola contaminado con hidrocarburos. Biotecnol Sustentabilidad 2016;1(1):82-5.

11.Marín Velásquez TD, Gota Díaz CN, Ortiz Pinto TC. Evaluación del extracto obtenido como lixiviado de fibra de coco (Cocos nucifera) como bioestimulante en la remediación de un suelo contaminado con petróleo. Enfoque UTE 2018;9(4):180-93. DOI: https://doi.org/10.29019/ enfoqueute.v9n4.303

12.Marín Velásquez TD. Extracto hidroalcohólico de cáscaras de naranja (Citrus sinensis) como bioestimulador en un suelo de sabana contaminado con petróleo. Ingeniería 2017;21(2):1-10.

111
13.Gómez W, Gaviria J, Cardona S. Evaluación de la bioestimulación frente a la atenuación natural y la bioaumentación en un suelo contaminado con una mezcla de gasolina-diésel. Dyna 2009;76(160):83-93.

14.ASTM D422-63(2007)e2, Standard Test Method for Particle-Size Analysis of Soils (Withdrawn 2016) [Internet]. ASTM International, West Conshohocken, PA; 2007 [citado 26 de octubre de 2019]. DOI: https://doi.org/10.1520/D0422-63R0 $\underline{7 \mathrm{E} 02}$

15.United States Environmental Protection Agency [Internet]. SW 846 Test Method 9071B: Nhexane extractable material (HEM) for sludge, sediment, and solid samples. Washington, DC: United States Environmental Protection Agency, c1998-2016; 1998. Recovered from: https:// www.epa.gov/sites/production/files/201512/documents/9071b.pdf

16. ASTM D4972-19, Standard Test Methods for $\mathrm{pH}$ of Soils [Internet]. ASTM International, West Conshohocken, PA; 2019 [citado 26 de octubre de 2019]. DOI: https://doi.org/10.1520/D4972-19

17.Marín Marin TD. Crecimiento de plantas de maíz (Zea mays) en un suelo contaminado con petróleo y remediado con extracto de cáscaras de naranja (Citrus sinensis). Enfoque UTE 2016;7(3):1-13. DOI: https://doi.org/10.29019/enfoqueute.v7n3. $\underline{101}$

18. Romero Saldaña M. Pruebas de bondad de ajuste a una distribución normal. Enferm Trab 2016; 6(3):105-14.

19.Fallas J. Análisis de varianza. Comparando tres o más medias [Internet]. Costa Rica: Universidad para la Cooperación Internacional; 2012. [citado 23 junio 2020]. ]. 54 p. Recuperado de: http:// www.ucipfg.com/Repositorio/MGAP/MGAP- 
05/BLOQUE-ACADEMICO/Unidad-2/comple mentarias/analisis de varianza 2012.pdf

20.Balzarini M, González LA, Tablada M, Casanoves F, Di Rienzo JA, Robledo CW. InfoStat Software Estadístico Manual del Usuario Versión 2008 [monografía en internet]. Córdova: Universidad Nacional de Córdoba; 2008. [acceso 15 de agosto de 2019]. Disponible en: https://www. researchgate.net/publication/283491340_Infostat manual del usuario

21.United State Department of Agriculture [Internet]. Soil Quality Test Kit Guide; 1999. [citado 20 agosto 2019]. Recuperado a partir de: https:// efotg.sc.egov.usda.gov/references/public/WI/Soil _Quality_Test_Kit_Guide.pdf

22.Kiatkittipong W, Phimsen S, Kiatkittipong K, Wongsakulphasatch S, Laosiripojana N, Assabumrungrat S. Diesel-like hydrocarbon production from hydroprocessing of relevant refining palm oil. Fuel Process Technol 2013;116:16-26. DOI: $\quad$ https://doi.org/10.1016/j.fuproc.2013.04. $\underline{018}$

23.Wang Y, Huang Y. Hydrogen isotopic fractionation of petroleum hydrocarbons during vaporization: implications for assessing artificial and natural remediation of petroleum contamination. Appl Geochem 2003;18(10);1641-51. DOI: https://doi.org/10.1016/s0883-2927(03)00076-3

24.Silvana CE, Martínez MA, Arocena MA. Estudio comparativo del agregado de enmiendas orgánicas e inorgánicas en procesos de biorremediación de suelos norpatagónicos contaminados con petróleo. Rev Soc Quím Peru 2014;80(4):251-61. DOI: https://doi.org/10.37761/rsqp.v80i4.177

25.Acuña AJ, Tonín NL, Díaz V, Pucci GN, Pucci $\mathrm{OH}$. Optimización de un sistema de biorremediación de hidrocarburos a escala de laboratorio. Ing Invest Tecnol 2012;13(1):105-12. DOI: https:// doi.org/10.22201/fi.25940732e.2012.13n1.011
26.Rodríguez Magda GE. Remoción de hidrocarburos totales en suelos contaminados con petróleo mediante residuos de cachaza y bagazo de caña de azúcar. UCV-Scientia 2017;9(1):59-66. DOI: https://doi.org/10.18050/RevUcv-Scientia.v9n1a6

27.Fernández C, Llobregat MJ, Bastidas H, Sien B. Influencia de la Eisenia foetida y de sustratos orgánicos como agentes bioestimulantes en la biodegradación de un suelo contaminado con petróleo pesado. Inf Tecnol 2009;20(5):19-30. DOI: https://doi.org/10.4067/S0718-07642009000500 $\underline{004}$

28. Buendía H. Biorremediación de suelos contaminados por hidrocarburos mediante el compost de aserrín y estiércol. Revista del Instituto de Investigación de la Facultad de Geología, Minas, Metalurgia y Ciencias Geográficas 2013;15(30):12330. DOI: https://doi.org/10.15381/iigeo.v15i30. $\underline{4101}$

29.Tanzi CD, Vian MA, Guinies C, Elmaataoui M, Chemat F. Terpenes as green solvents for extraction of oil from microalgae. Molecules 2012;17:8196-205. DOI: https://doi.org/10.3390/ molecules 17078196

30.Kumar SPJ, Prasad SR, Banerjee R, Agarwal DK, Kulkarni KS, Ramesh KV. Green solvents and technologies for oil extraction from oilseeds. Chem Cen J 2017;11(1):1-7. DOI: https://doi.org /10.1186/s13065-017-0238-8

31.Zeng WC, Zhang Z, Gao H, Jia LR, He Q. Chemical composition, antioxidant, and antimicrobial activities of essential oil from pine needle (Cedrus deodara). J Food Sci 2012;77(7):C8249. DOI: https://doi.org/10.1111/j.1750-3841.2012 $.02767 . \mathrm{x}$

32. Mitić ZS, Jovanović B, Jovanović SČ, Stojanović Radića ZZ, Mihajilov Krstev T, Javonovi'NM, et al. Essential oils of Pinus halepensis and P. heldreichii: Chemical composition, antimicrobial 
and insect larvicidal activity. Ind Crops Prod 2019;140: 111702. DOI: https://doi.org/10.1016 /j.indcrop.2019.111702

33.León Méndez G, Osorio Fortich MR, Martínez Useche SR. Comparación de dos métodos de extracción del aceite esencial de Citrus sinensis L. Rev Cubana Farm 2015;49(4):742-50.

34. Yañez Rueda X, Lugo Mancilla LL, Parada Parada DY. Estudio del aceite esencial de la cáscara de la naranja dulce (Citrus sinensis, variedad Valenciana) cultivada en Labateca (Norte de Santander, Colombia). Bistua 2007;5(1):3-8.

35.Tenorio Domínguez M. Flavonoides extraídos de la cascara de naranja tangelo (Citrus reticulata $\mathrm{x}$ Citrus paradisi) y su aplicación como antioxidante natural en el aceite vegetal sacha inchi (Plukenetia volubilis). Scientia Agropecuaria 2016;7(4):419-31. DOI: https://doi.org/10.17268/ sci.agropecu.2016.04.07

36.Iturbe Argüelles R. ¿Qué es la remediación? [Internet]. México: Dirección general de la divulgación de la ciencia; 2010 [citado 22-de octubre de 2019]. 24 p. Recuperado a partir de: http://www. dgdc.unam.mx/assets/cienciaboleto/cb_11.pdf
37.Irene Ortiz Bernad I, Sanz García J, Dorado Valiño M, Villar Fernández S. Técnicas de recuperación de suelos contaminados [Internet]. Madrid: Círculo de Innovación en tecnologías Medioambientales y Energía (CITME); 2007 [citado 15 de octubre de 2019]. 109 p. informe de vigilancia tecnológica vt 7. Recuperado a partir de: https://www.madrimasd.org/uploads/informacion idi/biblioteca/publicacion/doc/VT/vt6_tecnicas_r ecuperacion suelos contaminados.pdf

38.Corona Ramírez L, Iturbe Argüelles R. Atenuación natural en suelos contaminados con hidrocarburos. Ing Invest Tecnol 2005;6(2):119-26.

39.Romaniuk R, Brandt JF, Ríos PR, Giuffré L. Atenuación natural y remediación inducida en suelos contaminados con hidrocarburos. Cienc Suelo 2007;25(2):139-49.

Nota del Editor:

Journal of the Selva Andina Research Society (JSARS) se mantiene neutral con respecto a los reclamos jurisdiccionales publicados en mapas y afiliaciones institucionales. 